\title{
Variational Monte Carlo study of pentaquark states in a correlated quark model
}

\author{
Mark W. Paris \\ Theory Group, Thomas Jefferson National Accelerator Facility, 12000 Jefferson Avenue, MS 12H2, \\ Newport News, Virginia, 23606
}

\begin{abstract}
Accurate numerical solution of the five-body Schrödinger equation is effected via variational Monte Carlo. The spectrum is assumed to exhibit a narrow resonance with strangeness $S=+1$. A fully antisymmetrized and pair-correlated five-quark wave function is obtained for the assumed non-relativistic Hamiltonian which has spin, isospin, and color dependent pair interactions and many-body confining terms which are fixed by the non-exotic spectra. Gauge field dynamics are modeled via flux tube exchange factors. The energy determined for the ground states with $J^{\pi}=\frac{1}{2}^{-}\left(\frac{1}{2}^{+}\right)$is $2.22 \mathrm{GeV}(2.50 \mathrm{GeV})$. A lower energy negative parity state is consistent with recent lattice results. The short-range structure of the state is analyzed via its diquark content.
\end{abstract}

Keywords: pentaquark, quark model, quark correlations

PACS: 12.39.Jh,12.39.Pn,12.40.Yx,21.30.Fe,21.45.+v

A system of interacting, non-relativistic constituent quarks is the most simple, realistic model of hadronic systems. Solving the many-body Schrödinger equation to determine wave functions within this simple model is still a formidable task owing to the strong flavor, spin, and color dependence of the quark-quark interaction and traditionally requires some array of approximate methods to solve it. The controversial status of the recent evidence of a flavor exotic five-quark state warrants a careful treatment of this strongly interacting system. We have determined the wave function of five interacting constituent quarks in the flavor-exotic multiquark hadronic sector is solved using the variational Monte Carlo (VMC) technique [1]. This technique is known to yield upper bounds on the ground state energy accurate to the level of a few percent in light nuclei with the number of nucleons $A \leq 6$ [2].

Recent experimental evidence (for reviews see $[3,4]$ ) has revived interest in the multiquark flavor-exotic sector of the hadronic spectrum. Various model calculations have been reported (an overview is given in [5]) which study the existence of a strangeness $S=+1$ resonance, dubbed $\theta^{+}$, about $100 \mathrm{MeV}$ above threshold to nucleon-kaon decay with low mass of 1540 (2) $\mathrm{MeV}$ and possibly extremely narrow width of 0.9 (3) $\mathrm{MeV}$ [6]. Lattice results are available, including Refs. [7, 8]. High statistics photoproduction data of the reaction $\gamma p \rightarrow \bar{K}^{0} K^{+} n$ from the CLAS collaboration [9] sets an upper limit on the yield of the $\theta^{+}$relative to the $\Lambda^{*}(1520)$ yield at $0.2 \%$. No definitive structure in the $n K$-invariant mass spectrum is observed at $1540 \mathrm{MeV}$ in this experiment. No experimental information is available on the spin or parity of the state in any experiment done to date.

We study the $\theta^{+}$in the non-relativistic flux tube quark model with one-gluon exchange (OGE) and one-pion exchange (OPE) (between the light quarks). We take seriously the possibility that $\theta^{+}$is a narrow resonance and calculate its mass as a stable

CP842, Particles and Nuclei,

Seventeenth International Conference on Particles and Nuclei

edited by P. D. Barnes, M. D. Cooper, R. A. Eisenstein, H. van Hecke, and G. J. Stephenson

(C) 2006 American Institute of Physics 0-7354-0338-4/06/\$23.00 
TABLE 1. Variational energies of the states of pentaquark states in $\mathrm{GeV}$. The mass is reported as $M_{\theta^{+}}\left(n ; J_{4}^{\pi}\right)=\langle\hat{T}\rangle+$ $\langle\hat{V}\rangle-385.5(5) \mathrm{MeV}$. Statistical errors for $M_{\theta^{+}}$are $<5 \mathrm{MeV}$.

\begin{tabular}{c|ccccccc}
\hline$n ; J_{4}^{\pi}$ & $1 ; 1^{-}$ & $1 ; 1^{+}$ & $2 ; 1^{+}$ & $3 ; 0^{+}$ & $3 ; 1^{+}$ & $4 ; 0^{+}$ & $4 ; 1^{+}$ \\
\hline$M_{\theta^{+}}$ & 2.22 & 2.50 & 2.57 & 2.75 & 2.81 & 2.83 & 2.88 \\
$\langle\hat{T}\rangle$ & 1.68 & 2.13 & 2.02 & 2.03 & 2.00 & 1.92 & 1.90 \\
$\langle\hat{V}\rangle$ & 0.92 & 0.74 & 0.93 & 1.10 & 1.19 & 1.29 & 1.36
\end{tabular}

state with respect to strong interactions. The approach adopted here will be to work with a general, completely antisymmetric wave function and determine dynamically which flavor-spin-color-orbital (TSCL) structures are favored in the constituent quark model (CQM). The model Hamiltonian used in this work is fixed by the single hadron spectrum [10] and the six-quark (deuteron) properties [11]. It is given as a sum of kinetic energy, pair potentials for OGE and OPE, and confining terms. The two-body operator potentials for OGE (OPE) interactions are determined in the non-relativistic reduction of the tree level amplitude for $q q$ or $q \bar{q}$ scattering. We use a many-body confining interaction based on the "flux tube" model [12]. We consider flux tube topologies (there are three) for the $4 q \bar{q}$ states consistent with gauge invariance. Matrix elements of operators which connect different flux tube topologies are modeled by a quark position dependent factor which models the dynamics of the gauge fields [11].

The state vector for the five quarks, including two-body isospin, spin, color and spatial correlations is given by

$$
\left|\Psi_{5}\right\rangle=\mathscr{S} \prod_{i<j} \hat{F}_{i j}\left|\Phi_{5}\right\rangle
$$

where the symmetrized product of two-body correlation operators $\hat{F}_{i j}$ acts on an uncorrelated TSCL state, $\left|\Phi_{5}\right\rangle$. The state $\left|\Phi_{5}\right\rangle$ and, therefore, $\left|\Psi_{5}\right\rangle$ is completely antisymmetric under exchange of the coordinates of the $u$ and $d$ quarks.

We considered a large set of states obtained by considering states of total isospin $T=0, S=0,1$, and $L=0,1$ to yield states of negative and positive parity. The negative parity state $\left|1 ; 1^{-}\right\rangle$corresponds to the state of $4 q$ with positive parity, $\pi_{4}=+1$ with zero orbital angular momentum. The positive parity states, $\left|n=1, \ldots, 4 ; J_{4}^{+}\right\rangle$correspond to states of $4 q$ with negative parity and include one unit of orbital angular momentum. Here $J_{4}^{\pi}$ denotes the spin of the four light quarks, $J_{4}$ and the parity, $\pi$ of the pentaquark state.

The evaluation of expectation values of the variational wave function $\left|\Psi_{5}\right\rangle$ are effected via Monte Carlo integration of the 15 dimensional space $\mathbf{R}=\left(\mathbf{r}_{1}, \ldots, \mathbf{r}_{5}\right)$. Results for the variational energies of negative and positive parity states are shown in Table 1 . The mass of the state is reported as

$$
M_{\theta^{+}}\left(n ; J_{4}^{\pi}\right)=4 m_{q}+m_{s}+\langle\hat{T}\rangle+\langle\hat{V}\rangle-V_{0}(4 q \bar{q})
$$

where $\langle\hat{T}\rangle$ and $\langle\hat{V}\rangle$ are the variational kinetic and potential energies, respectively, for the states considered. The quark masses are taken as $m_{q}=313 \mathrm{MeV}, m_{s}=550 \mathrm{MeV}$ and $V_{0}$ is a constant fit to the nucleon mass. In reality, the true positive parity ground state is some admixture of the six positive parity states. However, the lowest energy state 
dominates since the states $\left|n ; J_{4}^{\pi}\right\rangle$ are orthonormal: applying the correlations as in Eq.(1) introduces off-diagonal elements which are quite small. States with $n \neq 1$ contribute $<10 \%$ to the true positive parity ground state.

Comparison of the CQM with lattice results at large pion mass is meaningful since the effects of chiral symmetry are negligible. We note that our negative parity state $\left|1 ; 1^{-}\right\rangle$ lies below the positive parity state $\left|1 ; 1^{+}\right\rangle$, consistent with the lattice data in Refs.[7,8]. This is true despite the fact that the positive parity state has much stronger attraction from OGE and OPE contributions, attributable to the high degree of symmetry of the $T S$ state (see Table 1). The $\langle\hat{V}\rangle$ of the negative parity state is $180 \mathrm{MeV}$ below the lowest positive parity state. Exciting one unit of orbital angular momentum on the other hand raises the kinetic energy significantly, about $450 \mathrm{MeV}$.

We have shown that within the non-relativistic flux tube CQM with OGE and OPE interactions the negative parity state is lower than the positive parity state by $\sim 280$ $\mathrm{MeV}$ when the $\theta^{+}$is assumed to be a narrow resonance. Though the higher symmetry of the lowest lying positive parity state significantly decrease its potential energy, its unit excitation of orbital angular momentum raises the energy above that of the negative parity state. Quark correlations are crucial in making these determinations and should not be ignored.

\section{ACKNOWLEDGMENTS}

The author would like to thank Jozef Dudek, Bob Wiringa, and Ross Young for helpful discussions. This work was supported by DOE contract DE-AC05-84ER40150 Modification No. M175, under which the Southeastern Universities Research Association (SURA) operates the Thomas Jefferson National Accelerator Facility.

\section{REFERENCES}

1. M. W. Paris, Phys. Rev. Lett. 95, 202002 (2005), nucl-th/ 0507061.

2. B. S. Pudliner, V. R. Pandharipande, J. Carlson, and R. B. Wiringa, Phys. Rev. Lett. 74, 4396-4399 (1995).

3. A. R. Dzierba, C. A. Meyer, and A. P. Szczepaniak, J. Phys. Conf. Ser. 9, 192-204 (2005), hep-ex/ 0412077.

4. K. H. Hicks, Prog. Part. Nucl. Phys. 55, 647-676 (2005), hep-ex/ 0504027.

5. M. Oka, Prog. Theor. Phys. 112, 1-19 (2004), hep-ph/ 0406211.

6. S. Eidelman, et al., Phys. Lett. B592, 1 (2004).

7. N. Mathur, et al., Phys. Rev. D70, 074508 (2004), hep-ph / 0406196.

8. B. G. Lasscock, et al., Phys. Rev. D72, 014502 (2005), hep-lat/ 0503008.

9. M. Battaglieri, D. Vita, et al. (2005), hep-ex/0510061.

10. M. W. Paris, Quantum Monte Carlo calculations of three and six-quark states, Ph.D. thesis, University of Illinois at Urbana-Champaign (2000).

11. M. W. Paris, and V. R. Pandharipande, Phys. Rev. C62, 015201 (2000), nucl-th/ 0002041.

12. N. Isgur, and J. Paton, Phys. Rev. D31, 2910 (1985). 